\title{
Functional Selectivity and Partial Efficacy at the Monoamine Transporters: A Unified Model of Allosteric Modulation and Amphetamine-Induced Substrate Release
}

\author{
Peter S. Hasenhuetl, ${ }^{1,2}$ Shreyas Bhat, ${ }^{2}$ Michael Freissmuth, and Walter Sandtner \\ Institute of Pharmacology, Gaston H. Glock Research Laboratories for Exploratory Drug Development, \\ Center of Physiology and Pharmacology, Medical University of Vienna, Vienna, Austria \\ Received October 2, 2018; accepted December 13, 2018
}

\section{ABSTRACT}

All clinically approved drugs targeting the plasmalemmal transporters for dopamine, norepinephrine, and serotonin act either as competitive uptake inhibitors or as amphetamine-like releasers. Monoamine transporter (MAT) ligands that allosterically affect MAT-mediated substrate uptake, release, or both were recently discovered. Their modes of action have not yet been explained in a unified framework. Here, we go beyond competitive inhibitors and classic amphetamines and introduce concepts for partial efficacy at and allosteric modulation of MATs. After we elaborate on a kinetic account for amphetamine action, we provide an explanation for partial release (i.e., the observation that some amphetamines are less efficacious than others in inducing monoamine efflux). We then elucidate mechanisms of allosteric inhibition and stimulation of MATs, which can be functionally selective for either substrate uptake or amphetamineinduced release. These concepts are integrated into a parsimonious kinetic framework, which relies exclusively on physiologic transport modes (without any deviation from an alternating access mechanism). The model posits cooperative substrate and $\mathrm{Na}^{+}$binding and functional selectivity by conformational selection (i.e., preference of the allosteric modulators for the substrate-loaded or substrate-free states of the transporter). Thus, current knowledge about the kinetics of monoamine transport is sufficiently detailed to provide a quantitative description of the releasing action of amphetamines, of substrate uptake, and of selective modulation thereof by allosteric modulators.

\section{Introduction}

The monoamines dopamine, serotonin, and norepinephrine are the most prominent neuromodulators in the human brain. These transmitters control the entire processing chain performed by the brain, from sensory input to behavioral output; perhaps the most prominent examples are the roles of dopamine in reinforcement learning and in voluntary movement, and the role of serotonin in the regulation of affective and emotional states. Because these transmitter systems are integral for normal brain function, it is not surprising that perturbations in brain monoamine signaling lead to many

This work was supported by the Austrian Science Fund/Fonds zur Förderung der Wissenschaftlichen Forschung [Grant P28090 (to W.S.)] and the Wiener Wissenschafts- und Technologie-Fonds [Grant LS17-026 (to M.F.)].

${ }^{1}$ Current affiliation: Centre for Neural Circuits and Behaviour, University of Oxford, Oxford, United Kingdom.

${ }^{2}$ P.S.H. and S.B. contributed equally to this work.

https://doi.org/10.1124/mol.118.114793. neuropsychiatric disorders. Consequently, most drugs used to treat these disorders interfere with monoamine signaling or homeostasis. They target the following: 1) neurotransmitter binding to their cognate receptors expressed in both preand postsynaptic neurons (most of these receptors are seven-transmembrane-spanning G protein-coupled receptors; Greengard, 2001); 2) neurotransmitter clearance from the extracellular space back into the presynaptic neuron by the plasmalemmal monoamine transporters (MATs), the serotonin transporter (SERT), the dopamine transporter (DAT), and the norepinephrine transporter (NET); 3) monoamine packaging into synaptic vesicles through vesicular monoamine transporters (VMATs); or 4) their degradation by the monoamine oxidases and the catechol- $O$-methyltransferase (see Alexander et al., 2015 for a comprehensive overview). Although clinically relevant drugs affecting the monoaminergic systems target this wide range of different targets, the efficacy spectrum of these drugs at the molecular

ABBREVIATIONS: DAT, dopamine transporter; MAT, monoamine transporter; NET, norepinephrine transporter; PAL-1045, (S)- $N$-ethyl-1-(2naphthyl)propan-2-amine; $\mathrm{PIP}_{2}$, phosphatitylinositol-4,5-bisphosphate; SERT, serotonin transporter; SRI-9804, N-(diphenylmethyl)-2-phenyl-4quinazolinamine; SRI-20040, N-(2,2-diphenylethyl)-2-phenyl-4-quinazolinamine; SRI-20041, $N$-(3,3-diphenylpropyl)-2-phenyl-4-quinazolinamine; SRI-29574, N-(2,2-diphenylethyl)-2-(imidazo[1,2-a]pyridin-6-yl)quinazolin-4-amine; SRI-30522, 2-([1,2,4]triazolo[1,5-a]pyridin-6-yl)-N-(2,2diphenylethyl)quinazolin-4-amine; VMAT, vesicular monoamine transporter. 
level has been narrow; the majority impair the function of their target proteins (i.e., they either inhibit the activity of enzymes or transporters or antagonize receptor-mediated signaling). In fact, inhibition/antagonism is currently the most prevalent form of pharmacological intervention.

Notable exceptions are drugs that bind to G protein-coupled receptors and to steroid hormone receptors; some of these drugs display partial efficacy and functional selectivity. Partial efficacy refers to the phenomenon in which ligands (referred to as partial agonists) do not elicit the same maximum response as full agonists; in the presence of a full agonist, they act as competitive antagonists. Functional selectivity (or biased agonism) arises from the fact that $\mathrm{G}$ protein-coupled receptors can use more than one signaling pathway: the ligand-bound receptor can display relative preference for one signaling pathway over the other. A related phenomenon is seen with nuclear transcription factors, which act as (hormone) receptors: their activity can be biased upon binding of some ligands. The conceptual framework that allows for understanding this complex pharmacology is not only of academic interest but also of potential clinical relevance (Michel and Charlton, 2018). In addition, the activity of G protein-coupled receptors can be modulated by ligands that occupy allosteric binding sites other than the canonical (orthosteric) agonist binding site: both negative and positive allosteric modulators of many $\mathrm{G}$ protein-coupled receptors have been identified but their therapeutic potential has not yet been exemplified (Christopoulos, 2014). However, ligandgated ion channels harbor binding sites, which are addressed by clinically relevant drugs (Christopoulos et al., 2014).

We argue that the pharmacology of MAT ligands is also more complex than represented by the current dichotomy, which categorizes them into two groups: 1) inhibitors block uptake of cognate neurotransmitters in a competitive manner (Kristensen et al., 2011), and 2) releaser drugs (most notably, amphetamine and its congeners) are MAT substrates (Sitte and Freissmuth, 2015). They are thus transported into the presynaptic neurons where they are exchanged for cognate neurotransmitters; this leads to nonvesicular monoamine release. Although these mechanisms of action differ considerably on the molecular level, they have the same consequence (i.e., an increase in neurotransmitter levels in the extracellular space). Moreover, at saturating concentrations, both ligand classes can completely abolish transporter-mediated substrate uptake. However, MAT ligands that also display attributes reminiscent of the concepts of partial efficacy and functional selectivity were recently identified: for instance, specific amphetamine congeners, termed "partial releasers," display reduced efficacy in inducing neurotransmitter efflux via MATs compared with traditional "full releasers" (Rothman et al., 2012; Sandtner et al., 2014; Bhat et al., 2017; Hasenhuetl et al., 2018). These actions can be viewed as analogous to partial agonism. In addition, functionally selective modulation of either substrate uptake or amphetamine-induced monoamine release has been reported, be it by exogenous (Rothman et al., 2015) or endogenous ( $\mathrm{Li}$ et al., 2017) allosteric modulators or by experimental manipulations (e.g., Kantor and Gnegy, 1998; Kantor et al., 2001; Khoshbouei et al., 2004; Steinkellner et al., 2012, 2014, 2015; Buchmayer et al., 2013; Kern et al., 2017). Moreover, the functionally selective allosteric modulator SRI20041 [N-(3,3-diphenylpropyl)-2-phenyl-4-quinazolinamine] also displays partial efficacy in inhibiting uptake when present at saturating concentrations; it is thus a partial inhibitor of substrate uptake (Rothman et al., 2015). Therefore, several compounds can neither be classified as "full" inhibitors nor as "full" releasers and their action cannot be accounted for by the currently held alternating access model of transmembrane transport without invoking additional conformational states. Here, we present a framework that provides a parsimonious explanation for atypical actions without digressing from the alternating access model, and we focus on the following topics:

1. Amphetamine-induced monoamine release: The currently available kinetic knowledge about the SERT is sufficient to account qualitatively and quantitatively for the releasing action of amphetamines when a cooperative binding model is used (Hasenhuetl et al., 2018). It is not necessary to invoke any amphetamine-specific transport modes such as a substrate-conducting pore (Kahlig et al., 2005; Rodriguez-Menchaca et al., 2012).

2. Partial release: This parsimonious model provides two explanations for the phenomenon that some amphetamine congeners are less efficacious than others in promoting efflux (Rothman et al., 2012; Sandtner et al., 2016; Bhat et al., 2017; Hasenhuetl et al., 2018).

3. Functional selectivity: It is possible to selectively modulate either monoamine uptake (Rothman et al., 2009; Li et al., 2017) or amphetamine-induced release (e.g., Kantor and Gnegy, 1998; Kantor et al., 2001; Khoshbouei et al., 2004; Fog et al., 2006; Sucic et al., 2010; Cremona et al., 2011; Steinkellner et al., 2012, 2014, 2015; Buchmayer et al., 2013; Pizzo et al., 2013; Hamilton et al., 2014; Moritz et al., 2015; Kern et al., 2017), yet a satisfactory molecular explanation for this phenomenon has remained elusive. We suggest that this can be accounted for by two simple kinetic features:

The two phenomena (uptake vs. release) are subject to two different rate-limiting reactions, which are kinetically distinct: monoamine uptake is rate limited by the slow return of the empty transporter to the outward-facing state (Bulling et al., 2012; Schicker et al., 2012; Hasenhuetl et al., 2016); monoamine efflux is rate limited by intracellular substrate exchange and subsequent outward translocation of the corresponding monoamine (Hasenhuetl et al., 2018).

Hence, relative preference to bind either the substrateloaded or substrate-free transporter (i.e., conformational selection) affects kinetically distinct partial reactions and can afford functional selectivity $(\mathrm{Li}$ et al., 2017). Importantly, this framework foregoes any deviation from an alternating access mechanism.

\section{Amphetamine-Induced Monoamine Release}

\section{Understanding MAT Pre-Steady-State Kinetics as a Prelude to Drug Discovery}

MATs face a dynamic environment in vivo; firing of monoaminergic neurons causes rapid changes in presynaptic membrane potential and concomitant dynamics in extracellular substrate concentrations. MATs clear released extracellular monoamines in only a few transport cycles and subsequently return to an $\mathrm{Na}^{+}$-bound outward-facing state. This occurs at 
rates of approximately $2 \mathrm{~s}^{-1}$ (Limberger et al., 1991; Erreger et al., 2008; Kristensen et al., 2011; Hasenhuetl et al., 2016). MAT turnover rates are slower than those of VMATs (approximately $6-8 \mathrm{~s}^{-1}$ ), which prevents accumulation of neurotransmitters in the cytosol. Moreover, the $\mathrm{Na}^{+} / \mathrm{K}^{+}$-ATPase prevents dissipation of both, $\mathrm{Na}^{+}$and $\mathrm{K}^{+}$gradients across neuronal plasma membranes. These concerted actions prevent cytosolic accumulation of monoamines and $\mathrm{Na}^{+}$. It is therefore clear that MATs never reach a steady state in vivo. Hence, novel therapeutic strategies should be executed with an underlying understanding of pre-steady-state kinetics of monoamine transport. We therefore briefly review basic principles of the MAT catalytic cycle under pre-steady-state conditions. The framework presented here is accompanied by a kinetic model of monoamine transport; this model is based on previously published models but to restrict the complexity of this discussion to a minimum, we 1) omitted the role of chloride and of $\mathrm{K}^{+} / \mathrm{H}^{+}$antiport in MAT physiology; 2) assumed the binding of only one $\mathrm{Na}^{+}$ion, although all three MATs are thought to have two $\mathrm{Na}^{+}$binding sites; and 3) neglected voltage-dependent processes and ion-conducting states. The reader is referred to the pertinent original work for a detailed account of these omitted factors (Erreger et al., 2008; Bulling et al., 2012; Schicker et al., 2012; Sandtner et al., 2014; Hasenhuetl et al., 2016; Bhat et al., 2017). A complete description of the model parameters is provided in the appendix in Hasenhuetl et al. (2018). They are dispensable for the current description, which focuses on the basic kinetic principles that afford atypical MAT pharmacology.

MATs are $\mathrm{Na}^{+}$-coupled monoamine symporters, which use the electrochemical gradient of $\mathrm{Na}^{+}$to import their cognate substrates. This is realized by an alternating access mechanism, where the substrate can be exposed either to the extracellular or the intracellular milieu when bound to the transporter, but not simultaneously (Jardetzky, 1966). Figure 1 shows an alternating access scheme of monoamine transport, which can be divided into four major parts. First, the outward-facing substrate-free transporter binds substrate and $\mathrm{Na}^{+}$; it harbors a substrate- and cosubstrate-binding core located halfway across the membrane. There, $\mathrm{Na}^{+}$and substrate have been suggested to form an interaction network, mediated by an aspartate residue (D79 in human DAT and D98 in human SERT) that interacts with the amine group of the substrate and with one of the $\mathrm{Na}^{+}$ions (Kristensen et al., 2011). Accordingly, it has been shown that $\mathrm{Na}^{+}$increases the affinity of substrate and vice versa (i.e., obeying a random, but cooperative binding scheme, as discussed below). Given the high external $\mathrm{Na}^{+}$concentrations found under physiologic conditions, the $\mathrm{Na}^{+}$-bound outward-facing species is thought to prevail, but the transporter can also bind substrate before $\mathrm{Na}^{+}$(Humphreys et al., 1994; Hasenhuetl et al., 2018). Second, only when $\mathrm{Na}^{+}$and substrate are bound simultaneously can the transporter isomerize to the inward-facing conformation. This entails several conformational rearrangements, yet it occurs at rates (50-100 $\left.\mathrm{s}^{-1}\right)$ that are at least one order of magnitude faster than the MAT uptake rates (1.5-3 $\mathrm{s}^{-1}$ ) (Erreger et al., 2008; Schicker et al., 2012; Hasenhuetl et al., 2016). Third, after isomerization to the inward-facing conformation, $\mathrm{Na}^{+}$and the substrate dissociate from the transporter; based on a large body of experimental data and molecular dynamics simulations, internal $\mathrm{Na}^{+}$is thought to dissociate before the substrate (Erreger et al., 2008; Malinauskaite et al., 2014; Hasenhuetl et al., 2016; Khelashvili et al., 2015; Razavi et al., 2017). This

\section{monoamine} uptake vs. efflux

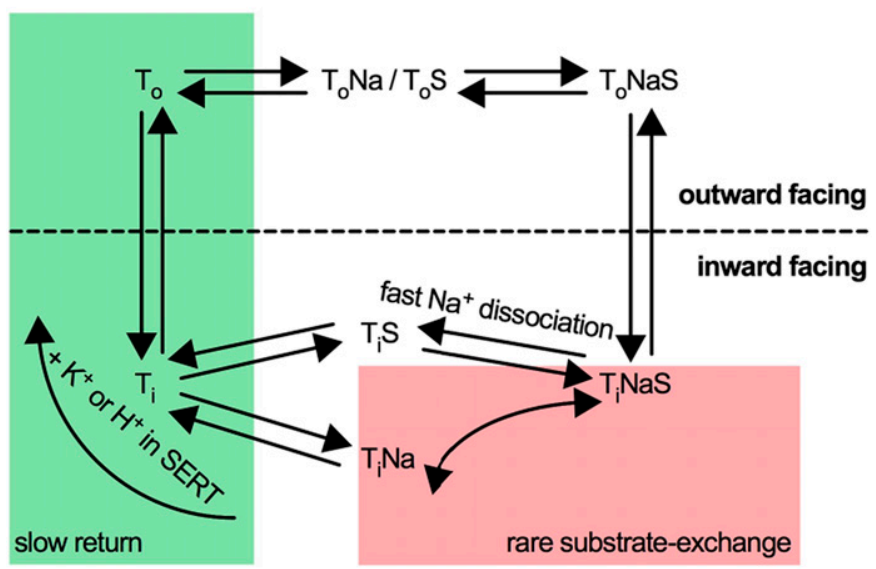

Fig. 1. Monoamine uptake and efflux are rate limited by different reactions. A simplified reaction scheme highlights different rate-limiting partial reactions. The rate-limiting reaction for substrate uptake is the return of the empty transporter from the inward-facing to the outwardfacing conformation (green area). In the majority of cases, internal $\mathrm{Na}^{+}$ dissociates before the substrate; occasionally, according to this model, substrate dissociates first, allowing for substrate exchange (red area). This process, or a partial reaction associated with it, is suggested to be rate limiting for amphetamine-induced monoamine release. Ti, empty inwardfacing transporter; TiNa, $\mathrm{Na}^{+}$-bound inward-facing conformation; TiNaS, $\mathrm{Na}^{+}$- and substrate-bound inward-facing conformation; TiS, substrate-bound inward-facing conformation; To, empty outward-facing transporter; ToNa, $\mathrm{Na}^{+}$-bound outward-facing conformation; ToNaS, $\mathrm{Na}^{+}$- and substrate-bound outward-facing conformation; ToS, substrate-bound outward-facing conformation.

dissociation occurs at a rapid rate (Hasenhuetl et al., 2016). Hence, neither the actual transition of the substrate into the cell nor the internal dissociation of $\mathrm{Na}^{+}$is rate limiting for substrate uptake. Fourth, the rate-limiting reaction determining substrate transport rates is the slow return step $\left(1.5-3 \mathrm{~s}^{-1}\right)$ of the substrate-free inward-facing transporter to the outward-facing conformation (green reaction in Fig. 1) (Mager et al., 1994; Bulling et al., 2012; Schicker et al., 2012; Hasenhuetl et al., 2016).

In essence, MATs import substrate by a rapid inward translocation of the substrate, which is followed by a slow return reaction of the substrate-free transporter (Erreger et al., 2008; Bulling et al., 2012; Schicker et al., 2012; Hasenhuetl et al., 2016). Importantly, it has been suggested that, rarely, substrate can dissociate from the $\mathrm{Na}^{+}-$and substrate-bound inward-facing conformation before the $\mathrm{Na}^{+}$ ion does (Hasenhuetl et al., 2018; red reaction in Fig. 1). In these situations, the transporter presents an $\mathrm{Na}^{+}$-bound substrate binding site to the inward-facing state and thus allows for exchange between the imported substrate and an intracellular substrate molecule; this substrate-exchange mode is thought to be the molecular basis of amphetamineinduced monoamine release (Sitte and Freissmuth, 2015), which is described below.

\section{Amphetamine-Triggered Monoamine Release Requires Cooperative Substrate and $\mathrm{Na}^{+}$Binding}

The prevailing theory of amphetamine action is the facilitated exchange diffusion model (Fischer and Cho, 1979; Sitte and Freissmuth, 2015): amphetamines are MAT substrates 
that release intracellular monoamines because they switch MAT function from the forward-transport to the substrateexchange mode. First, extracellular amphetamine binds to the outward-facing transporter, leading to transporter isomerization from the outward-facing to the inward-facing state. Second, the amphetamine subsequently dissociates from the inward-facing conformation, presenting a binding site for internal substrates. Third, in the presence of amphetamines, internal substrate accumulates due to depletion of synaptic vesicles (because amphetamines are also VMAT substrates) and to inhibition of monoamine-degrading enzymes such as the catechol-O-methyl transferase and the monoamine oxidases. Fourth, the higher prevalence of inward-facing substrate binding sites and the accumulation of internal substrate favor binding of intracellular monoamines, their subsequent outward translocation, and thus release.

Elevating the concentration of intracellular $\mathrm{Na}^{+}$augments amphetamine-induced efflux (Bönisch, 1986; Scholze et al., 2000; Khoshbouei et al., 2003; Kahlig et al., 2005). However, sequential binding of substrates and cosubstrates cannot account for the releasing action of amphetamines, because high internal $\mathrm{Na}^{+}$concentrations would impede dissociation of $\mathrm{Na}^{+}$and thus substrate exchange (Erreger et al., 2008; Hasenhuetl et al., 2016). Therefore, we recently developed a kinetic model of SERT in which we modeled binding not by a sequential order but by a random, yet cooperative, binding order (Hasenhuetl et al., 2018). It is important to note that the kinetic parameters used in this model were not chosen to specifically simulate amphetamine-induced substrate release. Instead, we constrained the parameters with robust kinetic measurements. These were derived from patch-clamp electrophysiology and were thus determined in real time (Hasenhuetl et al., 2016; Bhat et al., 2017) and verified by radioligand binding and uptake inhibition assays (Bhat et al., 2017). We then tested whether assuming only physiologic transport modes sufficed to explain the releasing action of amphetamines. The main concept behind the model was that cooperative binding connects the qualities of sequential and random binding and is therefore predicted to provide an account for both physiologic MAT function and the releasing action of amphetamines. The cooperative binding scheme posits that the affinities of $\mathrm{Na}^{+}$ and substrate are low when either of them bind alone, but that their affinities rise once they form a ternary complex. Indeed, the parsimonious alternating access model was sufficient to qualitatively and quantitatively recapitulate the releasing action of amphetamines (Hasenhuetl et al., 2018). Full releasers such as para-chloroamphetamine induce substrate release at rates on the order of several femtomoles per minute per cell in cells that heterologously express human SERT (Hilber et al., 2005; Seidel et al., 2005). Simulations using the cooperative binding model produced these release rates in a manner contingent on internal $\mathrm{Na}^{+}$concentrations (Hasenhuetl et al., 2018).

Interestingly, amperometric efflux measurements in DATexpressing outside-out patches revealed occasional bursts of dopamine release; these bursts (accounting for approximately $10 \%$ of total release, yet concentrated to a few milliseconds) were observed in addition to the slower bulk component of substrate efflux and could not be evoked by bath application of dopamine instead of amphetamine (Kahlig et al., 2005). The authors therefore proposed a mechanism of amphetamine action complementary to the alternating access model: substrate release via a substrate-conducting channel mode that is amphetamine specific and cannot be induced by the endogenous substrate (Kahlig et al., 2005).

The currently available alternating access model cannot recapitulate the amphetamine-specific bursts of dopamine release seen in amperometric recordings. It is possible that a substrate-conducting pore may be occasionally formed during the efflux process in a manner similar to the $\mathrm{Na}^{+}$-conducting state in SERT (Mager et al., 1994; Lin et al., 1996; Schicker et al., 2012). It will be interesting to test the relevance of these bursts compared with the bulk of transporter-mediated substrate efflux in vivo. However, given that only a minor fraction of efflux is accounted for by these bursts, it is not necessary to consider this amphetamine-specific mode (Adams and DeFelice, 2003; Kahlig et al., 2005; Rodriguez-Menchaca et al., 2012) to recapitulate the substrate release rates observed in efflux assays [compare Hasenhuetl et al. (2018) with Hilber et al. (2005) and Seidel et al. (2005)].

More importantly for the discussion that follows, adhering to the parsimonious alternating access mechanism allows for connecting: 1) substrate uptake, 2) the releasing action of "full" and "partial releasers," and 3) selective modulation thereof by allosteric modulators in a single framework without digressing from physiologic transport modes.

\section{Partial Release}

Some amphetamine congeners display a reduced efficacy in inducing monoamine release compared with "classical" amphetamines such as para-chloroamphetamine or 3,4-methylenedioxy$N$-methylamphetamine (Rothman et al., 2012; Sandtner et al., 2014). This lower rate of release is insurmountable by increasing substrate concentrations in a manner analogous to partial agonism at $\mathrm{G}$ protein-coupled receptors. These compounds have hence been termed "partial releasers." At least two mechanisms that explain this phenomenon can be envisaged.

First, the releaser may bind in two distinct binding modes, either as a releaser/substrate or as an inhibitor. Thus, assuming comparable binding kinetics of the two modes, increasing the concentration does not change the ratio between the two modes. The methylated derivative of 3,4-methylenedioxy- $N$-methylamphetamine (3,4-methylenedioxy$N, N$-dimethylamphetamine) has been suggested to bind in such a mode (Sandtner et al., 2014).

Second, the amphetamine may display a long dwell time at the transporter, which hinders exchange of the amphetamine with internal monoamine. The naphthyl-propan-amine PAL1045 [ $(S)$ - $N$-ethyl-1-(2-naphthyl)propan-2-amine] displays a high affinity to the transporter and a reduced efficacy to induce monoamine release (Rothman et al., 2012).

In the latter case, we recently showed that there are at least three factors that determine the efficacy of the releaser to induce monoamine efflux (Hasenhuetl et al., 2018). The first factor is the lipophilicity of the compound. The more lipophilic the releaser, the more likely it is to diffuse through the membrane and bind to the inward-facing conformation with increasing releaser concentrations (Sandtner et al., 2014). The second factor is the equilibrium affinity of the amphetamine to the transporter (Bhat et al., 2017). PAL-1045 has a high affinity to SERT and DAT, which has been shown by radioligand binding experiments. This high affinity translates into a longer dwell time at the transporter, which in turn prevents 
exchange of amphetamine and internal substrate, and thus blunts substrate release. This is also reflected by the nanomolar potency of PAL-1045 to induce release. The third factor is the extent of cooperativity between $\mathrm{Na}^{+}$and the amphetamine, which can contribute to differences in efficacy due to subsequent changes in the apparent affinity of the amphetamine for the transporter (Hasenhuetl et al., 2018). For instance, para-chloroamphetamine (a full releaser) may display a very low cooperativity compared with PAL-1045 (a partial releaser), which likely binds in a highly cooperative fashion. Using these simple features in the cooperative binding model, we could account for partial efficacy to induce release (Hasenhuetl et al., 2018): the experimentally observed release rates of PAL-1045 were on the order of $0.5 \mathrm{fmol} / \mathrm{min}$ per cell when a PAL-1045 concentration of $3 \mu \mathrm{M}$ was used. The model, the parameters of which were set to reproduce radioligand binding, uptake inhibition, and electrophysiological recordings only, produced release rates of $1 \mathrm{fmol} / \mathrm{min}$ per cell, which are thus in good agreement with the experimental data.

\section{Functional Selectivity: Allosteric Modulation of the Forward-Transport and Substrate-Exchange Modes}

\section{Kinetic Features That Afford Functional Selectivity}

It is possible to selectively modulate substrate uptake or amphetamine-induced monoamine release. In accordance to the concepts found in G protein-coupled receptors, we refer to this phenomenon as "functional selectivity." How can functional selectivity be achieved in MATs? The observation that, for instance, amphetamine-induced monoamine release can be blunted by experimental manipulations without affecting substrate uptake has raised doubts about whether amphetamine action could be explained by a simple alternating access model (e.g., see Schmitt et al., 2013). In contrast, we argue that a parsimonious alternating access mechanism (employing the cooperative binding scheme described above) is sufficient to explain this phenomenon if some simple kinetic aspects are respected. These kinetic aspects have been verified by experiments (Mager et al., 1994; Erreger et al., 2008; Bulling et al., 2012; Schicker et al., 2012; Hasenhuetl et al., 2016). The most important factor to explain functional selectivity is the observation that substrate uptake and release are rate limited by two distinct partial reactions within the MAT catalytic cycle: 1) substrate uptake is rate limited by the slow return of the empty transporter to the outward-facing conformation (Mager et al., 1994; Bulling et al., 2012; Schicker et al., 2012; Hasenhuetl et al., 2016); 2) monoamine efflux is rate limited by the (rare) intracellular exchange between amphetamine and substrate and subsequent outward translocation of the respective monoamine (Hasenhuetl et al., 2018). Moreover, these partial reactions are separated by a series of intracellular binding and unbinding events. Therefore, decelerating the transitions between substrate-loaded outward- and inwardfacing states will not affect uptake unless the transition rate is reduced to close to or below the rate of the substrate-free return step. Conversely, targeting the substrate-free return step will never affect amphetamine-induced release simply because this partial reaction is distinct from the one employed during amphetamine action (compare the green area with the red area in Fig. 1). Therefore, ligands that display conformational selection (i.e., the relative preference to bind either to the substrate-loaded or substrate-free transporter) can give rise to functional selectivity at the MATs (Li et al., 2017). Consequently, we emphasize that functional selectivity could not be achieved if substrate uptake were rate limited by the inward translocation of the substrate-loaded transporter. This is in contrast to the bacterial MAT homolog leucine transporter of Aquifex aeolicus, in which unlocking of the inner gate during substrate translocation may be the rate-limiting reaction for substrate uptake (Billesbølle et al., 2015). Examples and explanations for the different forms of allosteric modulation of MATs are provided in the following sections.

\section{Functionally Selective Modulation of Monoamine Release}

The selective modulation of amphetamine-induced monoamine release is currently the most prevalent form of functional selectivity in MATs found in the MAT literature. To our knowledge, there are no small-molecule compounds that can selectively modulate amphetamine-induced monoamine release. However, there exists a plethora of experimental manipulations that have been shown to selectively alter monoamine efflux induced by amphetamines. These include abolishing the function of the N terminus (Khoshbouei et al., 2004; Sucic et al., 2010; Kern et al., 2017) or C terminus (Rickhag et al., 2013) of MATs, depletion of membrane-enriched phosphatitylinositol4,5-bisphosphate $\left(\mathrm{PIP}_{2}\right)$ (Buchmayer et al., 2013), prevention of protein-protein interactions such as DAT with flotillin-1 (Cremona et al., 2011; Pizzo et al., 2013), and inhibition of MAT phosphorylation (Fog et al., 2006; Steinkellner et al., 2012, 2014, 2015; Pizzo et al., 2014; Moritz et al., 2015). These modifications do not impair substrate uptake but reduce or completely abrogate amphetamine-induced release. Moreover, activating the $\beta \gamma$ subunit of heterotrimeric $\mathrm{G}$ proteins was recently shown to be sufficient to induce dopamine release (Garcia-Olivares et al., 2017).

A notable example is $\mathrm{PIP}_{2}$ depletion. Reducing membrane $\mathrm{PIP}_{2}$ levels diminishes amphetamine-induced substrate release in vitro in both SERT and DAT (via the substrateexchange mode) with no detectable effect on substrate uptake (i.e., the forward-transport mode) (Buchmayer et al., 2013; Hamilton et al., 2014). The observation that amphetamineinduced dopamine release via DAT but not substrate uptake is contingent on $\mathrm{PIP}_{2}$ has been confirmed to be behaviorally relevant using Drosophila melanogaster as a model (Hamilton et al., 2014). This raises one important question: How can the two transport modes be differentially affected without violating the rules of microscopic reversibility? One explanation may be that $\mathrm{PIP}_{2}$ selectively lowers the activation barrier for the conformational rearrangements involving substrateloaded transporters. Indeed, molecular dynamics simulations suggested that the interaction of the DAT $\mathrm{N}$ terminus with $\mathrm{PIP}_{2}$ is important for the isomerization from the outwardfacing to the inward-facing state (Khelashvili et al., 2015). In addition, it may be critical for intracellular $\mathrm{Na}^{+}$release from the $\mathrm{Na}_{2}$ site and consequent substrate dissociation. Therefore, substrate uptake (i.e., the forward-transport mode) would not be impaired by blocking this interaction, because intracellular $\mathrm{Na}^{+}$and substrate release never become rate limiting for uptake. On the other hand, this may very well be the case for the substrate-exchange mode and thus the actions of amphetamines. 
The prediction that changing the activation barrier for substrate-loaded transporters affects the substrate-exchange mode (i.e., release), but not the forward-transport mode (i.e., uptake), has been tested experimentally in the case of an N-terminal mutant of SERT (Kern et al., 2017). The mutant $\triangle 32$-SERT displayed a normal turnover rate (and thus, normal substrate uptake), but the substrate-exchange mode (i.e., amphetamine-induced substrate release) was reduced. The simulations in Fig. 2 recapitulate this finding using the simple assumption that increasing the activation barrier for the transitions of the substrate-loaded transporter reduces efflux but not substrate uptake. Functional selectivity is lost only when we arbitrarily reduce the rate to values below the return rate; this can be seen in a decrease in substrate uptake.

We emphasize that this framework obeys an alternating access mechanism and therefore does not require any amphetamine-specific transport modes; it only requires that substrate uptake and release be rate limited by kinetically distinct partial reactions (e.g., see Fig. 1).

Specific inhibition of the substrate-exchange mode may be desirable in conditions in which substrate release causes disease but substrate uptake should be maintained. For example, the DAT coding variant Ala559Val, which has been linked to attention deficit hyperactivity disorder, was shown to support "anomalous dopamine efflux" (Mazei-Robison et al.,
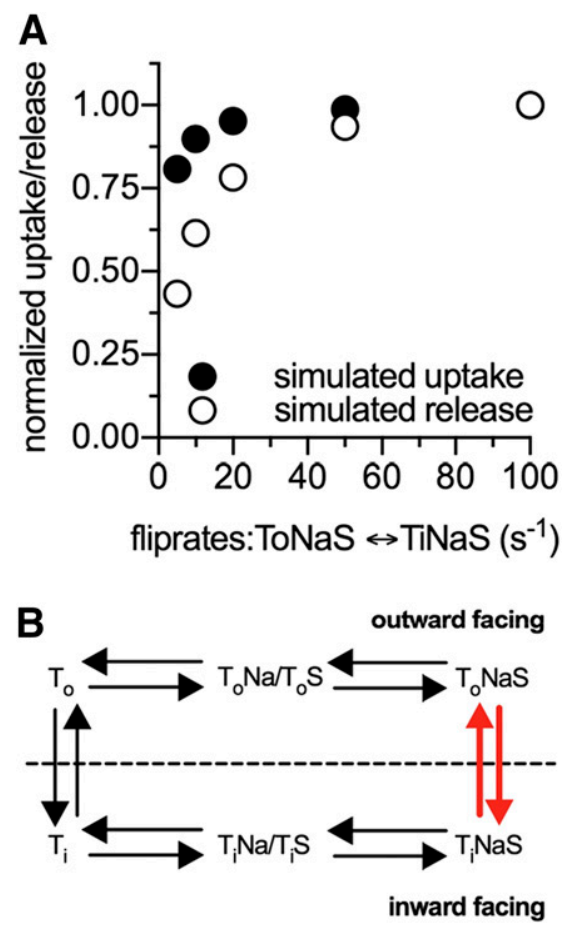

Fig. 2. The substrate translocation reaction is rate limiting for release but not for uptake. (A) Substrate uptake (closed circles) and amphetamineinduced substrate release (open circles) were simulated with a kinetic model and the transition rate between substrate-loaded outward-facing and inward-facing conformations was gradually reduced. The minimal reaction scheme of the catalytic cycle and affected rates (in red) is shown in (B). Ti, empty inward-facing transporter; $\mathrm{TiNa}, \mathrm{Na}^{+}$-bound inward-facing conformation; TiNaS, $\mathrm{Na}^{+}$- and substrate-bound inward-facing conformation; TiS, substrate-bound inward-facing conformation; To, empty outwardfacing transporter; ToNa, $\mathrm{Na}^{+}$-bound outward-facing conformation; ToNaS, $\mathrm{Na}^{+}$- and substrate-bound outward-facing conformation; ToS, substratebound outward-facing conformation.
2008; Bowton et al., 2010). That is, the transporter appeared to be in a state that favors nonvesicular dopamine release even in the absence of amphetamine and, notably, displayed an increased probability of dopamine release bursts in amperometric recordings compared with wild-type human DAT (Bowton et al., 2010). Specific targeting of the substrateexchange mode by an allosteric inhibitor is predicted to reduce this anomalous efflux but to have negligible effects on substrate uptake.

\section{Allosteric Modulation of Both Substrate Uptake and Release}

$\mathrm{Zn}^{2+}$ as an Example of a Functionally Unselective Allosteric Activator. Although the monoamine transport cycle has been resolved in considerable detail, kinetic knowledge on the molecular actions of synthetic allosteric modulators is still scarce. Fortunately, the DAT catalytic cycle is allosterically modulated by an endogenous ligand (namely, $\mathrm{Zn}^{2+}$; Norregaard et al., 1998). It is worth consulting $\mathrm{Zn}^{2+}$ as an instructive example, because its action on the DAT catalytic cycle has been deciphered to a large extent (Scholze et al., 2002; Meinild et al., 2004; Bjorklund et al., 2007; Pifl, et al., 2009; Li et al., 2015, 2017). For example, it can easily be washed in and removed from the bath solution during an experiment, the allosteric $\mathrm{Zn}^{2+}$ binding site is known (Norregaard et al., 1998; Loland et al., 1999; Stockner et al., 2013), and a $\mathrm{Zn}^{2+}$-nonresponsive mutant with otherwise indistinguishable transport function has been designed (Norregaard et al., 1998). $\mathrm{Zn}^{+}$binding stabilizes the outward-facing conformation of DAT (Loland et al., 2002; Stockner et al., 2013; Li et al., 2015). This potentiates both the forward-transport mode (i.e., DA uptake; Li et al., 2015 ) and the substrate-exchange mode (i.e., amphetamineinduced DA release; Meinild et al., 2004; Li et al., 2015). Importantly, the potentiating effect on substrate uptake is only evident when internal $\mathrm{Na}^{+}$concentrations are low $(\mathrm{Li}$ et al., 2015). If internal $\mathrm{Na}^{+}$concentrations rise during the experiment, the substrate-exchange mode dominates and the net effect of $\mathrm{Zn}^{2+}$ on uptake is inhibitory. Conversely, $\mathrm{Zn}^{2+}$ accelerates amphetamine-induced substrate release via DAT.

Most importantly, in the kinetic mechanism underlying the observed stimulation of DAT, only two features are required to explain all experimental findings (Li et al., 2015). First, $\mathrm{Zn}^{2+}$ binds the outward-facing state with higher affinity than the inward-facing state (Stockner et al., 2013; Li et al., 2015). Accordingly, either the dissociation rate is lower or the association rate is faster (or both) in the outward-facing conformation than in the inward-facing conformation. The rules of microscopic reversibility would be violated if all rates within the catalytic cycle were similar to the corresponding rates of the $\mathrm{Zn}^{2+}$-free transporter. Second, the transition of the substrate-free and substrate-loaded transporter must be accelerated to comply with microscopic reversibility. The former accelerates uptake, and the latter accelerates release (and blunts uptake at higher internal $\mathrm{Na}^{+}$). Both effects of $\mathrm{Zn}^{2+}$ have been confirmed by directly measuring the respective accelerated rates ( $\mathrm{Li}$ et al., 2015). Thus, $\mathrm{Zn}^{2+}$ is an allosteric activator that potentiates both the forward-transport mode (i.e., substrate uptake under initial rate conditions; Li et al., 2015) and the substrate-exchange mode (i.e., amphetamine action; Meinild et al., 2004; Li et al., 2015). Figure 3A shows simulations using a kinetic model, which can take an allosteric modulator into 
account. It is evident from the simulations that these assumptions suffice to account for the action of $\mathrm{Zn}^{2+}$ on the transport cycle of DAT. It is important to emphasize that $\mathrm{Zn}^{2+}$ has been shown to reduce dopamine uptake under conditions that favor intracellular $\mathrm{Na}^{+}$accumulation (Norregaard et al., 1998).

$\mathrm{Zn}^{2+}$ binding also increases the affinity of cocaine for DAT by stabilizing the outward-facing conformation. This can be explained by the stabilization of DAT in the outward-facing conformation. Interestingly, rigid adenine nucleoside derivatives were discovered that also decreased substrate uptake but increased radioligand binding affinity to DAT by an allosteric mechanism (Janowsky et al., 2016). We propose that these compounds display a mechanism of action comparable to that of $\mathrm{Zn}^{2+}$; that is, they stabilize the outward-facing conformation of DAT by an allosteric mechanism. It will be interesting to experimentally probe the action of these ligands on the pre-steady-state kinetics of DAT. If these compounds indeed display features comparable to $\mathrm{Zn}^{2+}$, they ought to be promising drug candidates for allosteric activation of DAT.

The endogenous $\mathrm{Zn}^{2+}$ binding site can also mediate functional selectivity: $\mathrm{Ni}^{2+}$ can also occupy the $\mathrm{Zn}^{2+}$ binding site of DAT. In contrast to $\mathrm{Zn}^{2+}$, however, $\mathrm{Ni}^{2+}$ specifically accelerates the forward-transport mode but does not have any appreciable effect on the substrate-exchange mode (see below).

\section{Simulated allosteric stimulation}
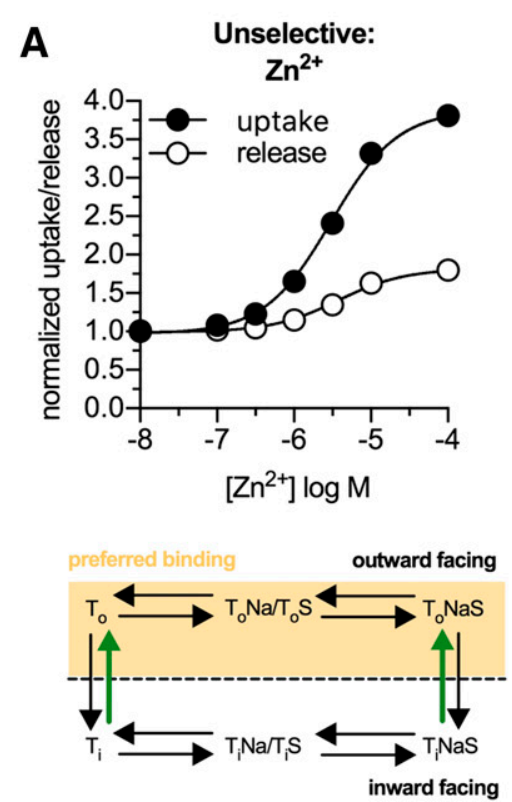
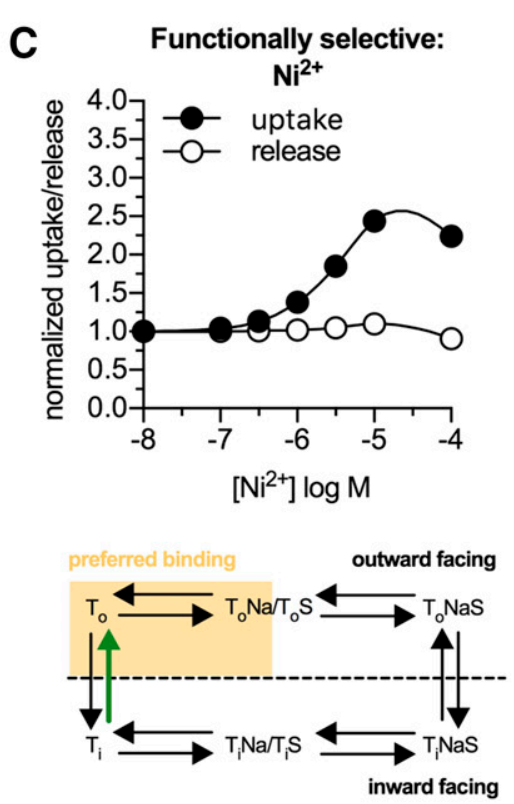

\section{Simulated allosteric inhibition}

\section{B}

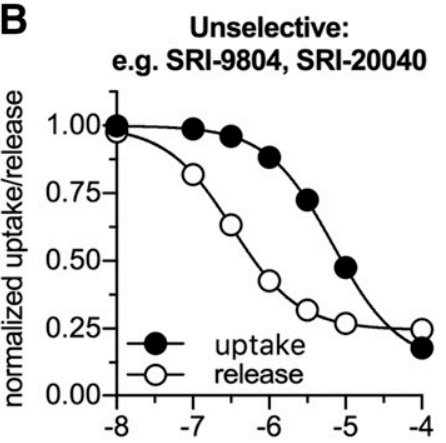

[SRI-20040] $\log M$

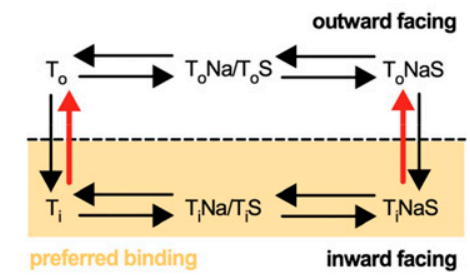

D Functionally selective: e.g. SRI-20041, SRI-29574, SRI-30522

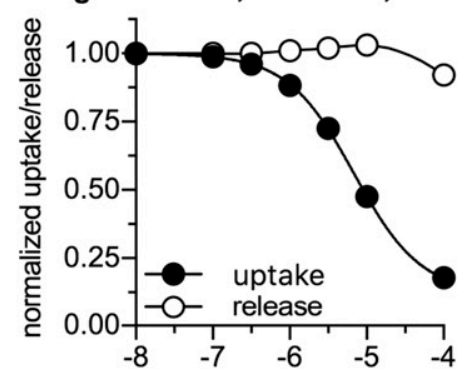

[SRI-20041] $\log M$

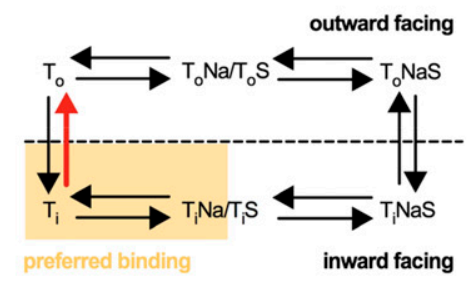

Fig. 3. Functional selectivity by conformational selection. (A) $\mathrm{Zn}^{2+}$ is a potentiator of both the forward-transport and substrate-exchange mode. Accordingly, it stimulates substrate uptake (closed circles) and amphetamine-induced substrate release (open circles). (B) SRI-20040 is a partial inhibitor of substrate uptake (closed circles) and release (open circles); it is thus the mirror image of $\mathrm{Zn}^{2+}$. Accordingly, we simulated its actions by modeling a higher affinity to the inward-facing conformation. (C) $\mathrm{Ni}^{+}$potentiates substrate uptake (closed circles) but does not have an effect on amphetamine-induced substrate release (open circles), even though it binds to the same binding site as $\mathrm{Zn}^{2+}$. (D) SRI-20041 is functionally selective for substrate uptake (closed circles) with no appreciable effect on substrate release (open circles); it is thus the mirror image of $\mathrm{Ni}^{2+}$. The reaction schemes in the individual panels illustrate the different mechanisms of action. The golden rectangles indicate the preferred species to which modulators bind within the catalytic cycle. Red arrows and green arrows indicate rates of partial reactions that need to be reduced or accelerated to maintain microscopic reversibility. Ti, empty inward-facing transporter; $\mathrm{TiNa}, \mathrm{Na}^{+}$-bound inward-facing conformation; TiNaS, $\mathrm{Na}^{+}$- and substrate-bound inwardfacing conformation; TiS, substrate-bound inward-facing conformation; To, empty outward-facing transporter; ToNa, $\mathrm{Na}^{+}$-bound outward-facing conformation; ToNaS, $\mathrm{Na}^{+}$and substrate-bound outward-facing conformation; ToS, substrate-bound outward-facing conformation. 
Allosteric, Partial Inhibition of Monoamine Uptake and Release. The quinazolinamines SRI-9804 [ $N$-(diphenylmethyl)2-phenyl-4-quinazolinamine] and SRI-20040 [ $N$-(2,2-diphenylethyl)2-phenyl-4-quinazolinamine] are allosteric modulators of DAT (Pariser et al., 2008; Rothman et al., 2009). They block substrate uptake and release and thus act on DAT in a manner opposite to $\mathrm{Zn}^{2+}$. Importantly, neither of these compounds fully abolished transporter activity at saturating concentrations. We therefore propose that the unselective partial inhibition of substrate uptake and release by the quinazolinamines is likely the consequence of conformational selection: we suggest that these ligands bind to an allosteric binding site. However, in contrast to $\mathrm{Zn}^{2+}$, which was shown to preferably bind to outward-facing transporters, we surmise that SRI-9804 and SRI-20040 bind with higher affinity to inward-facing transporters. The simulations in Fig. 3B reproduce the effects of SRI-9804 and SRI-20040 on DAT function.

\section{Functionally Selective Modulation of Uptake}

Thus far, we have discussed functionally selective modulation of amphetamine-induced monoamine release and unselective modulation of both uptake and release. There are also examples for ligands that selectively modulate substrate uptake with no appreciable effect on amphetamine action.

Allosteric Activation of Substrate Uptake. The endogenous $\mathrm{Zn}^{2+}$ binding site of DAT can also accept other bivalent cations; $\mathrm{Ni}^{2+}$, for instance, also binds to DAT. Interestingly, it has been shown that in contrast to $\mathrm{Zn}^{2+}, \mathrm{Ni}^{2+}$ selectively potentiates uptake by accelerating the slow rate-limiting step for completion of the forward-transport mode. $\mathrm{Ni}^{2+}$ does not modulate amphetamine-induced release (i.e., it does not affect the substrate-exchange mode). A kinetic model of DAT, which incorporated allosteric binding of transition metals (like $\mathrm{Zn}^{2+}$ or $\mathrm{Ni}^{2+}$ ), recapitulated the finding by modeling a higher affinity of $\mathrm{Ni}^{2+}$ not only to the outward-facing rather than the inward-facing state but, more specifically, to the outwardfacing substrate-free states of the transporter (Li et al., 2017). Hence, allosteric activation or inhibition can be achieved by favoring the outward-facing or the inward-facing conformation, respectively. Functional selectivity can be realized by also favoring either the substrate-free states (for uptake) or the substrate-loaded states (for release). In Fig. 3C, we show that an allosteric ligand, which favors binding to the substrate-free outward-facing conformation, enhances substrate uptake with no appreciable effect on amphetamineinduced monoamine release.

Allosteric activation of substrate uptake may provide a therapeutic benefit in the treatment of loss-of function mutations of DAT. For instance, a de novo mutation of human DAT linked to attention deficit hyperactivity disorder impairs the forward-transport and substrate-exchange mode (Hamilton et al., 2015). Strikingly, the function of this mutant transporter could be restored by application of $\mathrm{Zn}^{2+}$. Thus, a compound that mimics the action of $\mathrm{Zn}^{2+}$ on DAT ought to correct the deficiency.

Allosteric Inhibition of Substrate Uptake. The quinazolinamine SRI-20041 and more recently identified compounds such as SRI-29574 [N-(2,2-diphenylethyl)-2-(imidazo[1,2-a]pyridin6-yl)quinazolin-4-amine] and SRI-30522 [2-([1,2,4]triazolo[1,5-a]pyridin-6-yl)- $N$-(2,2-diphenylethyl)quinazolin-4-amine] selectively block uptake but not release (Rothman et al., 2015). This observation is reminiscent of the effect of nitric oxide on DAT
(Cao and Reith, 2002). Other quinazolinamines (SRI-9804 and SRI-20040) act as conformationally unselective partial inhibitors (Pariser et al., 2008; Rothman et al., 2009). Interestingly, SRI-29574 and SRI-30522 fail to affect radioligand binding to DAT, suggesting that they bind to a substrate-free species of the transporter. To date, there has been no kinetic framework to explain this phenomenon. We propose that the actions of SRI-20041 and its congeners can be parsimoniously explained by binding of the modulator to the inward-facing substratefree state in an allosteric fashion, thus only affecting the left (green) branch of the reaction scheme in Fig. 1 (Pariser et al., 2008; Rothman et al., 2015). Figure 3D shows a simulation of this prediction: uptake is impaired but release is not affected when the allosteric modulator prefers binding to the inwardfacing substrate-free state. Given that this binding mechanism does not include the substrate-bound state, we expect that this would also reproduce the negligible effect on radioligand binding. Electrophysiological recordings are predicted to reveal that under conditions that favor the forwardtransport mode (physiologic ion gradients), the rate-limiting step in the transport cycle would be reduced as a consequence of the stabilization of the inward-facing conformation. However, when high internal $\mathrm{Na}^{+}$concentrations are employed, relaxation from the inward-facing to the outward-facing state via the substrate-exchange mode would be indistinguishable from conditions without SRI-20041.

Partial inhibition is likely to have a milder side-effect profile compared with competitive inhibitors: it merely reduces the rate of uptake, but it does not abrogate transport. It is therefore conceivable that SRI-20041 can serve as a template to develop other functionally selective MAT modulators, including potentiators and partial inhibitors of the forwardtransport mode.

\section{Conclusions}

There has been substantial progress in the discovery and characterization of allosteric modulators, a kinetic framework for which we present here. Current knowledge about the kinetics of monoamine transport is sufficiently detailed to formulate a realistic model that can account for the releasing action of amphetamines, partial release, and selective modulation of substrate uptake and release. This model requires the following features: 1) a cooperative binding scheme is needed; 2) uptake and release are rate limited by two kinetically distinct reactions; and 3) relative preference for either the substrate-loaded or the substrate-free transporter can afford functional selectivity without any deviation from an alternating access mechanism.

Interestingly, selective modulation can be brought about even by binding to the same binding site: $\mathrm{Ni}^{2+}$ is more selective for the forward-transport mode than $\mathrm{Zn}^{2+}$ in spite of binding to the same site. This justifies the prediction that a collection of allosteric modulators can be identified based on a prototypical ligand; SRI-20041, SRI-29574, and SRI-30522 qualify as lead candidates. We hope that the framework presented here is useful for a rational approach to identifying and classifying new allosteric modulators of MAT function.

\section{Authorship Contributions}

Participated in research design: Hasenhuetl, Bhat, Freissmuth, Sandtner. 
Performed data analysis: Hasenhuetl, Bhat, Sandtner.

Wrote or contributed to the writing of the manuscript: Hasenhuetl, Bhat, Freissmuth, Sandtner.

\section{References}

Adams SV and DeFelice LJ (2003) Ionic currents in the human serotonin transporter reveal inconsistencies in the alternating access hypothesis. Biophys $J \mathbf{8 5}$ 1548-1559.

Alexander SP, Kelly E, Marrion N, Peters JA, Benson HE, Faccenda E, Pawson AJ, Sharman JL, Southan C, Buneman OP, et al.; CGTP Collaborators (2015) The Concise Guide to Pharmacology 2015/16: overview. $\mathrm{Br} J$ Pharmacol 172: $5729-5743$.

Bhat S, Hasenhuetl PS, Kasture A, El-Kasaby A, Baumann MH, Blough BE, Sucic S, Sandtner W, and Freissmuth M (2017) Conformational state interactions provide clues to the pharmacochaperone potential of serotonin transporter partial substrates. J Biol Chem 292:16773-16786.

Billesbølle CB, Krüger MB, Shi L, Quick M, Li Z, Stolzenberg S, Kniazeff J, Gotfryd K, Mortensen JS, Javitch JA, et al. (2015) Substrate-induced unlocking of the inner gate determines the catalytic efficiency of a neurotransmitter:sodium symporter. $J$ Biol Chem 290:26725-26738.

Bjorklund NL, Volz TJ, and Schenk JO (2007) Differential effects of $\mathrm{Zn}^{2+}$ on the kinetics and cocaine inhibition of dopamine transport by the human and rat dopamine transporters. Eur $J$ Pharmacol 565:17-25.

Bönisch H (1986) The role of co-transported sodium in the effect of indirectly acting sympathomimetic amines. Naunyn Schmiedebergs Arch Pharmacol 332:135-141.

Bowton E, Saunders C, Erreger K, Sakrikar D, Matthies HJ, Sen N, Jessen T, Colbran RJ, Caron MG, Javitch JA, et al. (2010) Dysregulation of dopamine transporters via dopamine D2 autoreceptors triggers anomalous dopamine efflux associated with attention-deficit hyperactivity disorder. $J$ Neurosci 30:6048-6057.

Buchmayer F, Schicker K, Steinkellner T, Geier P, Stübiger G, Hamilton PJ, Jurik A Stockner T, Yang JW, Montgomery T, et al. (2013) Amphetamine actions at the serotonin transporter rely on the availability of phosphatidylinositol-4,5-bisphosphate. Proc Natl Acad Sci USA 110:11642-11647.

Bulling S, Schicker K, Zhang YW, Steinkellner T, Stockner T, Gruber CW, Boehm S, Freissmuth M, Rudnick G, Sitte HH, et al. (2012) The mechanistic basis for noncompetitive ibogaine inhibition of serotonin and dopamine transporters. J Biol Chem 287:18524-18534.

Cao BJ and Reith ME (2002) Nitric oxide inhibits uptake of dopamine and N-methyl4-phenylpyridinium (MPP+) but not release of MPP+ in rat $\mathrm{C} 6$ glioma cells expressing human dopamine transporter. Br J Pharmacol 137:1155-1162.

Christopoulos A (2014) Advances in G protein-coupled receptor allostery: from function to structure. Mol Pharmacol 86:463-478.

Christopoulos A, Changeux JP, Catterall WA, Fabbro D, Burris TP, Cidlowski JA, Olsen RW, Peters JA, Neubig RR, Pin JP, et al. (2014) International Union of Basic and Clinical Pharmacology. XC. Multisite pharmacology: recommendations for the nomenclature of receptor allosterism and allosteric ligands. Pharmacol Rev 66 918-947.

Cremona ML, Matthies HJ, Pau K, Bowton E, Speed N, Lute BJ, Anderson M, Sen N, Robertson SD, Vaughan RA, et al. (2011) Flotillin-1 is essential for PKC-triggered endocytosis and membrane microdomain localization of DAT. Nat Neurosci 14 469-477.

Erreger K, Grewer C, Javitch JA, and Galli A (2008) Currents in response to rapid concentration jumps of amphetamine uncover novel aspects of human dopamine transporter function. J Neurosci 28:976-989.

Fischer JF and Cho AK (1979) Chemical release of dopamine from striatal homogenates: evidence for an exchange diffusion model. J Pharmacol Exp Ther 208. 203-209.

Fog JU, Khoshbouei H, Holy M, Owens WA, Vaegter CB, Sen N, Nikandrova Y, Bowton E, McMahon DG, Colbran RJ, et al. (2006) Calmodulin kinase II interacts with the dopamine transporter $\mathrm{C}$ terminus to regulate amphetamine-induced reverse transport. Neuron 51:417-429.

Garcia-Olivares J, Baust T, Harris S, Hamilton P, Galli A, Amara SG, and Torres GE (2017) G $\beta \gamma$ subunit activation promotes dopamine efflux through the dopamine transporter. Mol Psychiatry 22:1673-1679.

Greengard P (2001) The neurobiology of slow synaptic transmission. Science 294: $1024-1030$

Hamilton PJ, Belovich AN, Khelashvili G, Saunders C, Erreger K, Javitch JA, Sitte HH, Weinstein H, Matthies HJG, and Galli A (2014) PIP2 regulates psychostimulant behaviors through its interaction with a membrane protein. Nat Chem Biol 10:582-589.

Hamilton PJ, Shekar A, Belovich AN, Christianson NB, Campbell NG, Sutcliffe JS, Galli A, Matthies HJ, and Erreger K (2015) $\mathrm{Zn}(2+)$ reverses functional deficits in a de novo dopamine transporter variant associated with autism spectrum disorder. Mol Autism 6:8.

Hasenhuetl PS, Bhat S, Mayer FP, Sitte HH, Freissmuth M, and Sandtner W (2018) A kinetic account for amphetamine-induced monoamine release. J Gen Physiol 150:431-451.

Hasenhuetl PS, Freissmuth M, and Sandtner W (2016) Electrogenic binding of intracellular cations defines a kinetic decision point in the transport cycle of the human serotonin transporter. J Biol Chem 291:25864-25876.

Hilber B, Scholze P, Dorostkar MM, Sandtner W, Holy M, Boehm S, Singer EA and Sitte HH (2005) Serotonin-transporter mediated efflux: a pharmacological analysis of amphetamines and non-amphetamines. Neuropharmacology $\mathbf{4 9}$ $811-819$

Humphreys CJ, Wall SC, and Rudnick G (1994) Ligand binding to the serotonin transporter: equilibria, kinetics, and ion dependence. Biochemistry 33:9118-9125.

Janowsky A, Tosh DK, Eshleman AJ, and Jacobson KA (2016) Rigid adenine nucleoside derivatives as novel modulators of the human sodium symporters for dopamine and norepinephrine. J Pharmacol Exp Ther 357:24-35.
Jardetzky O (1966) Simple allosteric model for membrane pumps. Nature 211: 969-970.

Kahlig KM, Binda F, Khoshbouei H, Blakely RD, McMahon DG, Javitch JA and Galli A (2005) Amphetamine induces dopamine efflux through a dopamine transporter channel. Proc Natl Acad Sci USA 102:3495-3500.

Kantor L and Gnegy ME (1998) Protein kinase C inhibitors block amphetaminemediated dopamine release in rat striatal slices. J Pharmacol Exp Ther 284: $592-598$.

Kantor L, Hewlett GH, Park YH, Richardson-Burns SM, Mellon MJ, and Gnegy ME (2001) Protein kinase $\mathrm{C}$ and intracellular calcium are required for amphetaminemediated dopamine release via the norepinephrine transporter in undifferentiated PC12 cells. J Pharmacol Exp Ther 297:1016-1024.

Kern C, Erdem FA, El-Kasaby A, Sandtner W, Freissmuth M, and Sucic S (2017) The $\mathrm{N}$ terminus specifies the switch between transport modes of the human serotonin transporter. J Biol Chem 292:3603-3613.

Khelashvili G, Stanley N, Sahai MA, Medina J, LeVine MV, Shi L, De Fabritiis G, and Weinstein $\mathrm{H}$ (2015) Spontaneous inward opening of the dopamine transporter is triggered by PIP2-regulated dynamics of the N-terminus. ACS Chem Neurosci 6 : 1825-1837.

Khelashvili G, Schmidt SG, Shi L, Javitch JA, Gether U, Loland CJ, and Weinstein H (2016) Conformational dynamics on the extracellular side of LeuT controlled by $\mathrm{Na}+$ and $\mathrm{K}+$ ions and the protonation state of Glu290. J Biol Chem 291: 19786-19799.

Khoshbouei H, Sen N, Guptaroy B, Johnson L, Lund D, Gnegy ME, Galli A and Javitch JA (2004) N-terminal phosphorylation of the dopamine transporter is required for amphetamine-induced efflux. PLoS Biol 2:E78.

Khoshbouei H, Wang H, Lechleiter JD, Javitch JA, and Galli A (2003) Amphetamineinduced dopamine efflux. A voltage-sensitive and intracellular $\mathrm{Na}+$-dependent mechanism. J Biol Chem 278:12070-12077.

Kristensen AS, Andersen J, Jørgensen TN, Sørensen L, Eriksen J, Loland CJ, Strømgaard K, and Gether U (2011) SLC6 neurotransmitter transporters: structure, function, and regulation. Pharmacol Rev 63:585-640.

Li Y, Hasenhuetl PS, Schicker K, Sitte HH, Freissmuth M, and Sandtner W (2015) Dual Action of $\mathrm{Zn} 2+$ on the Transport Cycle of the Dopamine Transporter. J Biol Chem 290:31069-31076.

Li Y, Mayer FP, Hasenhuetl PS, Burtscher V, Schicker K, Sitte HH, Freissmuth M, and Sandtner W (2017) Occupancy of the zinc-binding site by transition metals decreases the substrate affinity of the human dopamine transporter by an allosteric mechanism. $J$ Biol Chem 292:4235-4243.

Limberger N, Trout SJ, Kruk ZL, and Starke K (1991) "Real time" measurement of endogenous dopamine release during short trains of pulses in slices of rat neostriatum and nucleus accumbens: role of autoinhibition. Naunyn Schmiedebergs Arch Pharmacol 344:623-629.

Lin F, Lester HA, and Mager S (1996) Single-channel currents produced by the serotonin transporter and analysis of a mutation affecting ion permeation. Biophys J 71:3126-3135.

Loland CJ, Norregaard L, and Gether U (1999) Defining proximity relationships in the tertiary structure of the dopamine transporter. Identification of a conserved glutamic acid as a third coordinate in the endogenous $\mathrm{Zn}(2+)$-binding site. J Biol Chem 274:36928-36934.

Loland CJ, Norregaard L, Litman T, and Gether U (2002) Generation of an activating $\mathrm{Zn}(2+)$ switch in the dopamine transporter: mutation of an intracellular tyrosine constitutively alters the conformational equilibrium of the transport cycle. Proc Natl Acad Sci USA 99:1683-1688.

Malinauskaite L, Quick M, Reinhard L, Lyons JA, Yano H, Javitch JA, and Nissen P (2014) A mechanism for intracellular release of $\mathrm{Na}+$ by neurotransmitter/sodium symporters. Nat Struct Mol Biol 21:1006-1012.

Mager S, Min C, Henry DJ, Chavkin C, Hoffman BJ, Davidson N, and Lester HA (1994) Conducting states of a mammalian serotonin transporter. Neuron 12 $845-859$.

Mazei-Robison MS, Bowton E, Holy M, Schmudermaier M, Freissmuth M, Sitte HH, Galli A, and Blakely RD (2008) Anomalous dopamine release associated with a human dopamine transporter coding variant. J Neurosci 28:7040-7046.

Meinild AK, Sitte HH, and Gether U (2004) Zinc potentiates an uncoupled anion conductance associated with the dopamine transporter. $J$ Biol Chem 279: 49671-49679.

Michel MC and Charlton SJ (2018) Biased agonism in drug discovery-is it too soon to choose a path? Mol Pharmacol 93:259-265.

Moritz AE, Rastedt DE, Stanislowski DJ, Shetty M, Smith MA, Vaughan RA, and Foster JD (2015) Reciprocal phosphorylation and palmitoylation control dopamine transporter kinetics. J Biol Chem 290:29095-29105.

Norregaard L, Frederiksen D, Nielsen EO, and Gether U (1998) Delineation of an endogenous zinc-binding site in the human dopamine transporter. $E M B O J \mathbf{1 7}$ 4266-4273.

Pariser JJ, Partilla JS, Dersch CM, Ananthan S, and Rothman RB (2008) Studies of the biogenic amine transporters. 12. Identification of novel partial inhibitors of amphetamine-induced dopamine release. J Pharmacol Exp Ther 326:286-295.

Pifl C, Wolf A, Rebernik P, Reither H, and Berger ML (2009) Zinc regulates the dopamine transporter in a membrane potential and chloride dependent manner. Neuropharmacology 56:531-540.

Pizzo AB, Karam CS, Zhang Y, Ma CL, McCabe BD, and Javitch JA (2014) Amphetamine-induced behavior requires CaMKII-dependent dopamine transporter phosphorylation. Mol Psychiatry 19:279-281.

Pizzo AB, Karam CS, Zhang Y, Yano H, Freyberg RJ, Karam DS, Freyberg Z, Yamamoto A, McCabe BD, and Javitch JA (2013) The membrane raft protein Flotillin-1 is essential in dopamine neurons for amphetamine-induced behavior in Drosophila. Mol Psychiatry 18:824-833.

Razavi AM, Khelashvili G, and Weinstein H (2017) A Markov state-based quantitative kinetic model of sodium release from the dopamine transporter. Sci Rep 7: 40076 . 
Rickhag M, Owens WA, Winkler MT, Strandfelt KN, Rathje M, Sørensen G, Andresen B, Madsen KL, Jørgensen TN, Wörtwein G, et al. (2013) Membranepermeable C-terminal dopamine transporter peptides attenuate amphetamineevoked dopamine release. J Biol Chem 288:27534-27544.

Rodriguez-Menchaca AA, Solis E Jr., Cameron K, and De Felice LJ (2012) S(+)amphetamine induces a persistent leak in the human dopamine transporter: molecular stent hypothesis. Br J Pharmacol 165:2749-2757.

Rothman RB, Ananthan S, Partilla JS, Saini SK, Moukha-Chafiq O, Pathak V, and Baumann MH (2015) Studies of the biogenic amine transporters 15. Identification of novel allosteric dopamine transporter ligands with nanomolar potency. $J$ Pharmacol Exp Ther 353:529-538.

Rothman RB, Dersch CM, Ananthan S, and Partilla JS (2009) Studies of the biogenic amine transporters. 13. Identification of "agonist" and "antagonist" allosteric modulators of amphetamine-induced dopamine release. J Pharmacol Exp Ther 329:718-728.

Rothman RB, Partilla JS, Baumann MH, Lightfoot-Siordia C, and Blough BE (2012) Studies of the biogenic amine transporters. 14. Identification of low-efficacy "partial" substrates for the biogenic amine transporters. J Pharmacol Exp Ther 341:251-262. Sandtner W, Schmid D, Schicker K, Gerstbrein K, Koenig X, Mayer FP, Boehm S, Freissmuth M, and Sitte HH (2014) A quantitative model of amphetamine action on the 5-HT transporter. Br J Pharmacol 171:1007-1018.

Sandtner W, Stockner T, Hasenhuetl PS, Partilla JS, Seddik A, Zhang YW, Cao J, Holy M, Steinkellner T, Rudnick G, et al. (2016) Binding mode selection determines the action of ecstasy homologs at monoamine transporters. Mol Pharmacol 89:165-175.

Schicker K, Uzelac Z, Gesmonde J, Bulling S, Stockner T, Freissmuth M, Boehm S, Rudnick G, Sitte HH, and Sandtner W (2012) Unifying concept of serotonin transporter-associated currents. J Biol Chem 287:438-445.

Schmitt KC, Rothman RB, and Reith ME (2013) Nonclassical pharmacology of the dopamine transporter: atypical inhibitors, allosteric modulators, and partial substrates. J Pharmacol Exp Ther 346:2-10.

Scholze P, Zwach J, Kattinger A, Pifl C, Singer EA, and Sitte HH (2000) Transportermediated release: a superfusion study on human embryonic kidney cells stably expressing the human serotonin transporter. J Pharmacol Exp Ther 293:870-878.
Scholze P, Nørregaard L, Singer EA, Freissmuth M, Gether U, and Sitte HH (2002) The role of zinc ions in reverse transport mediated by monoamine transporters. $J$ Biol Chem 277:21505-21513.

Seidel S, Singer EA, Just H, Farhan H, Scholze P, Kudlacek O, Holy M, Koppatz K, Krivanek P, Freissmuth M, et al. (2005) Amphetamines take two to tango: an oligomer-based counter-transport model of neurotransmitter transport explores the amphetamine action. Mol Pharmacol 67:140-151.

Sitte HH and Freissmuth M (2015) Amphetamines, new psychoactive drugs and the monoamine transporter cycle. Trends Pharmacol Sci 36:41-50.

Steinkellner T, Montgomery TR, Hofmaier T, Kudlacek O, Yang JW, Rickhag M, Jung G, Lubec G, Gether U, Freissmuth M, et al. (2015) Amphetamine action at the cocaine- and antidepressant-sensitive serotonin transporter is modulated by $\alpha$ CaMKII. J Neurosci 35:8258-8271.

Steinkellner T, Mus L, Eisenrauch B, Constantinescu A, Leo D, Konrad L, Rickhag M, Sørensen G, Efimova EV, Kong E, et al. (2014) In vivo amphetamine action is contingent on $\alpha$ CaMKII. Neuropsychopharmacology 39:2681-2693.

Steinkellner T, Yang JW, Montgomery TR, Chen WQ, Winkler MT, Sucic S, Lubec G Freissmuth M, Elgersma Y, Sitte HH, et al. (2012) $\mathrm{Ca}(2+) /$ calmodulin-dependent protein kinase II $\alpha$ ( $\alpha \mathrm{CaMKII})$ controls the activity of the dopamine transporter: implications for Angelman syndrome. J Biol Chem 287:29627-29635.

Stockner T, Montgomery TR, Kudlacek O, Weissensteiner R, Ecker GF, Freissmuth $\mathrm{M}$, and Sitte HH (2013) Mutational analysis of the high-affinity zinc binding site validates a refined human dopamine transporter homology model. PLoS Comput Biol 9:e1002909.

Sucic S, Dallinger S, Zdrazil B, Weissensteiner R, Jørgensen TN, Holy M, Kudlacek O, Seidel S, Cha JH, Gether U, et al. (2010) The N terminus of monoamine transporters is a lever required for the action of amphetamines. J Biol Chem 285:10924-10938.

Address correspondence to: Michael Freissmuth, Institute of Pharmacology, Medical University of Vienna, Waehringerstrasse 13a, A-1090 Vienna, Austria. E-mail: michael.freissmuth@meduniwien.ac.at 